\title{
ACERCA DE LA HIDRONEFROSIS SINTOMÁTICA EN LA GESTANTE.
}

\author{
Pedro Navalón Verdejo, Francisco Sánchez Ballester, Yoni Pallas Costa, José Antonio Cánovas \\ Ivorra, Felipe Ordoño Domínguez, Joaquín Juan Escudero, Luis De la Torre Abril y Macarena \\ Ramos de Campos.
}

Servicio de Urología. Consorcio Hospital General Universitario. Valencia. España.

\begin{abstract}
Resumen.- OBJETIVO La hidronefrosis durante el embarazo es un fenómeno frecuente que afecta al 90\% de las gestantes. Aunque habitualmente es asintomática, en un escaso porcentaje de pacientes se manifiesta clínicamente, precisando tratamiento. Con este estudio pretendemos poner de manifiesto nuestra experiencia en el diagnóstico y tratamiento de la uropatía obstructiva sintomática en la mujer embarazada.
\end{abstract}

MÉTODOS: Evaluamos retrospectivamente los resultados obtenidos en 162 gestantes afectas de hidronefrosis sintomática atendidas en nuestro servicio durante los últimos doce años.

RESULTADOS: El tratamiento conservador fue eficaz en la gran mayoría de las pacientes, precisándose en 39 de ellas medidas mas agresivas; así, en 35 casos se realizó colocación de catéter doble J, en 2 pacientes nefrostomía percutánea y en otras 2 se practicó extracción ureteroscópica de litiasis ureteral.

CONCLUSIONES: La etiología mas frecuente de hidronefrosis sintomática durante el embarazo la ha constituido la compresión por el útero grávido seguida de la litiasis ureteral. Consideramos de elección el tratamiento conservador, reservando el cateterismo con doble J o la nefrostomía percutánea para casos refractarios. La ureteroscopia constituye una nueva alternativa diagnóstica y terapéutica cuando fallan otras medidas menos agresivas.

Palabras clave: Embarazo. Hidronefrosis. Catéter doble J. Nefrostomía percutánea.

Summary.- OBJECTIVES: Hydronephrosis is common during pregnancy, affecting $90 \%$ of pregnant women. It is usually asymptomatic, but a small percentage of patients show clinical symptoms requiring treatment. We want to report our experience in the diagnosis and treatment of symptomatic obstructive uropathy in pregnant women.

METHODS: We retrospectively evaluate the results obtained in 162 pregnant women suffering symptomatic hydronephrosis who received care at our department over the last 12 years.

RESULTS: Conservative treatment was effective in most patients, 39 patients required more aggressive therapy. Thirty-five patients underwent insertion of a double J catheter, 2 patients percutaneous nephrostomy, and another 2 ureteroscopy and extraction of ureteral lithiasis.

CONCLUSIONS: The most frequent etiology of symptomatic hydronephrosis during pregnancy is external compression by 
the gravid uterus, followed by ureteral lithiasis. We consider conservative treatment as the treatment of choice, leaving ureteral double J catheter insertion or percutaneous nephrostomy for the refractory cases. Ureteroscopy is a new diagnostic and therapeutic option when other less aggressive measures fail.

Keywords: Pregnancy. Hydronephrosis. Double J catheter. Percutaneous nephrostomy.

\section{INTRODUCCIÓN}

La hidronefrosis durante el embarazo es un fenómeno frecuente que afecta al $90 \%$ de las gestantes $(1,2)$. Habitualmente aparece en la segunda mitad de la gestación, tratándose por lo general de una ectasia leve o moderada, indolora, que no interfiere la funcionalidad renal y que predomina en el lado derecho y en primigrávidas $(2,3)$.

En cuanto a las causas que intervienen en su desarrollo parecen estar implicados diversos factores, tales como la compresión extrínseca provocada por el aumento de volumen de las venas ováricas y del útero gravídico así como el efecto miorrelajante de la progesterona. Por otra parte, la dextrorrotación uterina y la protección relativa del uréter izquierdo por el colon sigmoide explicarían la mayor predisposición a la hidronefrosis derecha $(4,5)$. Asimismo, el incremento de la diuresis, pequeñas litiasis y otros factores no reconocidos pueden provocar descompensación de la función ureteral, convirtiendo la hidronefrosis en sintomática y por tanto susceptible de tratamiento; situación que afecta al 0.2-3\% de las gestantes (1).

Durante el embarazo, diferentes entidades patológicas pueden manifestarse clínicamente de forma similar a la crisis renoureteral, lo que puede llevar a un diagnóstico erróneo y un tratamiento inadecuado. Así, tanto la apendicitis como la colelitiasis pueden producir dolor en zonas distintas de las habituales como consecuencia del desplazamiento de los órganos abdominales por el útero, produciendo signos clínicos equívocos. De la misma forma, el desprendimiento de placenta y otras patologías obstétrico-ginecológicas también deben ser consideradas. No obstante, las causas urológicas (pielonefritis, hidronefrosis sintomática y litiasis) son las que mas frecuentemente producen dolor abdominal agudo en la gestante (4).

Aunque la presencia de hidronefrosis durante el embarazo es muy frecuente, sólo un escaso porcentaje de gestantes desarrollarán una dilatación renoureteral patológica que puede provocar dolor en el flanco y a veces deterioro de la función renal. Asimismo, como la presencia de bacteriuria está favorecida, puede propiciar el desarrollo de un cuadro séptico en el contexto de una uropatía obstructiva (6).

\section{MATERIAL Y MÉTODOS}

Presentamos nuestra experiencia durante los últimos doce años en el tratamiento de 162 gestantes atendidas en nuestro servicio por hidronefrosis sintomática. La mayor parte de las pacientes, 96 (59\%), fueron multíparas, siendo el tiempo medio de gestación de 32'4 semanas (rango 17 - 35).

El cuadro clínico de presentación predominante fue de dolor lumboabdominal de tipo cólico en 138 pacientes $(85 \%)$, mientras que en las 24 restantes (15\%), se manifestó clínicamente en forma de pielonefritis aguda.

La valoración urológica consistió en anamnesis detallada, hemograma y bioquímica sanguínea, sedimento urinario, urinocultivo y ecografía abdominal. En ningún caso realizamos exploración radiológica.

Un total de 123 pacientes (76\%) fueron controladas con tratamiento conservador (medidas posturales, analgésicos y/o antibióticos). En las 39 restantes $(24 \%)$, en las que fallaron estas medidas conservadoras se procedió a la colocación de catéter "doble J" en 35 pacientes, nefrostomía percutánea ecodirigida bajo anestesia local en dos casos y extracción endoscópica de cálculo urinario bajo anestesia raquídea con ureterorrenoscopio rígido en otras dos pacientes.

El seguimiento tras la instrumentación consistió fundamentalmente en la realización de sedimento urinario y urinocultivo quincenal, así como control ecográfico dentro de las 24 horas siguientes al procedimiento y posteriores controles ultrasonográficos de periodicidad variable entre 7 y 30 días (media 22 días).

\section{RESULTADOS}

Cuando las medidas conservadoras fallaron, en todos los casos se intentó como primera medida terapéutica la cateterización con "doble J" del sistema excretor dilatado, lo cual se realizó con éxito en 35 pacientes $(90 \%)$. En las 4 restantes $(10 \%)$ no se consiguió colocar el catéter "doble J"; en dos de 
ellas por litiasis infranqueable en tercio inferior ureteral que se resolvieron con extracción endoscópica con ureteroscopio rígido, y en otras dos pacientes, ambas con litiasis impactada en uréter lumbar, se realizó nefrostomía percutánea y ulterior litotricia por ondas de choque postparto.

Tras la colocación del catéter "doble J", 26 pacientes $(74 \%)$ presentaron mejoría de la sintomatología en las 24 horas siguientes a la manipulación; otras 2 pacientes $(6 \%)$ presentaron ligeras molestias lumbares así como síntomas irritativos vesicales persistentes que se controlaron con la administración de analgésicos hasta la retirada del catéter. Las 7 pacientes restantes $(20 \%)$ continuaron presentando tras la instrumentación polaquiuria, estranguria y hematuria por un periodo máximo de 8 días, siendo tratadas con anticolinérgicos y analgésicos.

El control ecográfico postcateterismo fue realizado dentro de las 24 horas siguientes al mismo, y en todos los casos se confirmó la desaparición o disminución del grado de ectasia. El catéter fue retirado a las 2 ó 3 semanas del parto realizándose nuevo examen ultrasonográfico al mes de la retirada del catéter.

En las 24 pacientes que debutaron como pielonefritis aguda, el urinocultivo fue positivo para Escherichia coli en 21 casos (88\%) y en las 3 restantes (12\%) se aisló Proteus mirabilis.

Durante el seguimiento, 26 pacientes (16\%) presentaron sedimento urinario patológico con intensa piuria y bacteriuria significativa, 16 de ellas asintomáticas, mientras que 10 presentaron clínica irritativa miccional; todas ellas fueron tratadas indistintamente con antibioterapia (fosfomicina o $\beta$-lactámicos).

La etiología litiásica de la obstrucción únicamente se demostró en 57 pacientes (35\%); en 2 de ellas se practicó extracción endoscópica durante la gestación, en otras 43 pacientes se produjo la expulsión espontánea del cálculo (29 durante la gestación y 14 tras la retirada del catéter), mientras que en los 12 casos restantes se practicó litotricia endoscópica o por ondas de choque tras el parto.

\section{DISCUSIÓN}

La uropatía obstructiva durante el embarazo es un hallazgo frecuente que afecta al $90 \%$ de las gestantes $(1,2)$; se desarrolla a partir de la sexta semana de gestación y desaparece entre la cuarta y sexta semanas postparto $(3,4)$. Aunque generalmente es asintomática, puede manifestarse clínicamente en el 0 '2 - 3\% de las pacientes (1).

La acción de la progesterona sobre el músculo liso produciendo miorrelajación y disminución de la peristalsis, se considera la causante de esta ectasia fisiológica a partir de la $6^{\mathrm{a}}-10^{\mathrm{a}}$ semanas de embarazo (4). Sin embargo, en fases mas evolucionadas del embarazo, la dilatación se limita al segmento ureteral por encima de la pelvis, lo que hace mas probable la intervención de otros factores en su desarrollo, tales como la compresión ureteral por el útero gravídico, la dilatación de las venas ováricas y ciertas situaciones especiales como el polihidramnios y el embarazo gemelar $(2,4)$. La dextrorrotación del útero, el cruce con las venas ováricas dilatadas o la compresión de la cabeza fetal se relacionan con una mayor incidencia de hidronefrosis en el lado derecho, proporcionando el sigma cierto grado de protección en el lado izquierdo (4). Asimismo, algunos autores propugnan la etiología mecánica compresiva como la principal causa de hidronefrosis fisiológica basándose en la ausencia de casos descritos en gestantes con riñones pélvicos $o$ en portadoras de conducto ileal. Igualmente, el hecho de que la dilatación ureteral ocurre más a menudo y progresa más rápidamente después de la $21^{\underline{a}}$ semanas de gestación sostiene la teoría de la compresión por el útero grávido (3).

La diuresis intensa, infecciones, litiasis y otros factores no conocidos pueden descompensar la hidronefrosis convirtiéndola en sintomática y por lo tanto susceptible de tratamiento.

En lo que se refiere a la etiología litiásica, durante la gestación se produce una hipercalciuria absortiva fisiológica causada por la producción placentaria de 1,25 dihidrocolecalciferol y supresión de hormona paratiroidea; todo lo cual aumenta la excreción de calcio; pudiendo favorecer la formación de cálculo. Por otra parte se incrementan la diuresis, así como la filtración de citrato, magnesio y glucosaminglicanos que inhiben la litogénesis. Así, aunque existe sobresaturación de oxalato cálcico, no se suele encontrar cristaluria. Este equilibrio entre factores predisponentes e inhibidores de la litogénesis parece ser la causa de que la incidencia de las litiasis no se vea afectada por la gestación $(7,8)$. De hecho, un elevado porcentaje de las gestantes con cólico renal tienen historia previa de litiasis, afectando con mayor frecuencia a mujeres multíparas que primíparas en una proporción 3:1 $(9,10)$. No obstante, el cólico nefrítico se considera la causa mas frecuente de hospitalización durante el embarazo por dolor abdominal $(11,12)$ siendo el parto prematuro la complicación obstétrica mas común que puede provocar (13). 
Durante la gestación, las estructuras retroperitoneales se suelen mantener fijas, pero los órganos intraabdominales se movilizan por la compresión del útero grávido pudiendo provocar cuadros con clínica similar al cólico nefrítico como es el caso de la patología apendicular y vesicular $(4,9)$. Así, en la experiencia de Sthoters y cols (10), el $28 \%$ de las pacientes con litiasis urinaria se diagnosticaron erróneamente de apendicitis, diverticulitis o desprendimiento de placenta. Asimismo, las mujeres embarazadas experimentan frecuentemente nauseas, vómitos, dolor dorsal, polaquiuria y disuria, constituyendo un desafío importante para cualquier urólogo la interpretación de estos síntomas en asociación con hidronefrosis.

Las técnicas habituales de diagnóstico por la imagen presentan limitaciones durante el embarazo debido al potencial efecto perjudicial de las radiaciones sobre el feto, siendo conocida su capacidad de inducir carcinogénesis, teratogénesis y mutaciones $(14,15)$. En este contexto, aunque algunas publicaciones propugnan la utilización del urograma excretor de una sola radiografía $(4,16)$, la mayor parte de los autores revisados desaconsejan su utilización, ya que una única dosis de radiación parece ser mas periudicial que varias exposiciones menores (15), habiéndose descrito también la posibilidad de ciertos cambios funcionales o bioquímicos ante la exposición a bajos niveles de irradiación (14). Así mismo, la dificultad para la interpretación de las proyecciones por la hidronefrosis fisiológica, el retraso en la eliminación de contraste, así como la superposición de eventuales litiasis sobre el esqueleto fetal y útero, disminuyen su rendimiento y la hacen desaconsejable; más aún si consideramos el riesgo potencial que supone la exposición a radiaciones y las posibles reacciones alérgicas al medio de contraste. De igual modo, la pielografía retrograda la la que se suma el potencial riesgo de sepsis) y la TAC por su alto nivel de irradiación deben ser evitadas en la medida de lo posible.

En la actualidad, el diagnóstico de dilatación de la vía urinaria se realiza con facilidad mediante el uso de la ecografía sin exponer el feto a riesgos innecesarios; sin embargo resulta difícil diferenciar la hidronefrosis fisiológica de la obstructiva, con una sensibililidad del $34 \%$ y especificidad del $86 \%$ para la detección de litiasis en las pacientes sintomáticas (10). Se ha intentado optimizar el rendimiento de la ecografía por diferentes autores. Así, algunos aconsejan la realización de un renograma en pacientes sintomáticas con un diámetro de la pelvis superior a $17 \mathrm{~mm}(17)$; sin embargo, otros han descrito ectasias mayores en pacientes asintomáticas (18). La carencia de eyaculación ureteral ecográficamente también se ha utilizado para el diagnóstico de obstrucción, obteniéndose una alta sensibilidad y especificidad (18), aunque otros estudios aportan varios casos de pacientes asintomáticas con ausencia de eyaculación ureteral (19). Otra aplicación del ultrasonido es la vía transvaginal, que se ha mostrado útil para el diagnóstico de litiasis en la porción más distal del uréter (20). También se ha empleado la ecografía dopplercolor, cuya utilidad real es controvertida $(21,22)$.

Otra técnica a considerar es la urografía por $R M$, procedimiento del que no se han descrito efectos perjudiciales para el feto, con una sensibilidad del $100 \%$ para diferenciar la dilatación fisiológica de la obstructiva y con buena correlación con el renograma excretor. No obstante, se trata de una técnica cara y no disponible en la mayoría de los centros, aunque recientes publicaciones la consideran el método diagnóstico de elección en aquellos casos en los que la ecografía no proporciona suficientes datos de valoración (23).

El tratamiento debe estar dirigido al control de los síntomas, para lo que es conveniente una hidratación adecuada, reposo, analgésicos y antibióticos cuando coexiste infección. En nuestra experiencia, también consideramos de gran valor terapéutico las medidas posturales, recomendando el decúbito lateral sobre el lado opuesto al del riñón dilatado en un intento de disminuir la compresión ureteral por el útero grávido.

En lo que se refiere al empleo de antiinflamatorios no esteroideos (AINE) durante el ambarazo, el ácido acetil-salicílico no se debe utilizar ya que disminuye la contractilidad uterina, provoca cierre precoz del conducto arterioso y aumenta el riesgo de sangrado antes y después del parto tanto para el feto como para la madre (24). Otros AINE cuyo empleo se desaconseja por el riesgo de cierre del conducto arterioso y oligohidramnios son el naproxeno e ibuprofeno, aunque se pueden emplear con seguridad durante menos de 48 horas. Respecto al metamizol, aunque no se han realizado estudios adecuados y bien controlados en humanos, no se han demostrado efectos adversos con su utilización en mujeres embarazadas; no obstante debe evitarse su administración durante el primer trimestre de gestación por falta de estudios sobre teratogenia así como durante las seis últimas semanas del embarazo por la inhibición de la síntesis de prostaglandinas que produce, aceptándose su uso tan solo bajo estricta vigilancia clínica; por tanto, la administración del metamizol durante otros periodos del embarazo solo se acepta en caso de ausencia de alternativas terapéuticas mas seguras (24). Los AINE recomendados son el paracetamol y propoxifeno ya que no se han descrito efectos tóxicos 
sobre el feto y consiguen una analgesia media que generalmente es suficiente para controlar los cuadros de dolor agudo. Cuando esta pauta de analgesia moderada no es suficiente para el control de la sintomatología, la administración de opiáceos a bajas dosis, como el sulfato de morfina y la meperidina, puede ser de gran valor terapéutico, aunque hay que tener en consideración las graves consecuencias que su utilización prolongada puede producir al feto (adicción a narcóticos, retraso del crecimiento intrauterino y parto prematuro). Asimismo, la administración de codeína se debe limitar al $2^{\circ}$ y 3 er trimestre con bajo riesgo para el feto siempre que se emplee a bajas dosis (24). En cuanto a los antibióticos recomendados, las penicilinas y cefalosporinas se consideran de elección por su amplio espectro e inocuidad para el feto $(3,14)$. Una alternativa segura son la fosfomicina y los macrólidos, con la excepción del estolato de eritromicina que produce colestasis y hepatotoxicidad en el feto $(13,24)$.

El tratamiento conservador es eficaz en la gran mayoría de casos, permitiendo posponer el diagnóstico etiológico hasta una vez finalizada la gestación. En un porcentaje que varía entre el $7 \%$ y el $35 \%$ según diversas publicaciones revisadas 11,2 , $6,10)$ no existe una mejoría sintomática a pesar de las medidas conservadoras, precisando en ocasiones la realización de procedimientos invasivos, tales como la cateterización ureteral, nefrostomía percutánea $\circ$ ureteroscopia con litotricia o sin ella. Estas técnicas mas agresivas las consideramos indicadas en casos de cólico refractario, sepsis, obstrucción de riñón solitario y cuando motivos psicológicos o sociales las justifiquen.

En nuestra experiencia, la proporción de pacientes que precisaron instrumentación fue del $24 \%$. No obstante, el porcentaje real debió ser menor ya que obviamente hubo pacientes tratadas directamente por el ginecólogo, de las que no poseemos registro.

En caso de tener que recurrir a la instrumentación, nosotros consideramos de elección como primera medida terapéutica la derivación urinaria interna con catéteres doble J, ya que se trata de un método de fácil ejecución sin necesidad de anestesia, con escasa morbilidad, permitiendo un drenaje duradero de la vía urinaria $(1,2,6)$. No obstante, aunque nosotros no hayamos constatado ninguna complicación con su utilización, la técnica no esta exenta de ellas, siendo la mas frecuentemente documentada la incrustación, para cuya prevención se han propuesto la hidratación, restricción de calcio y profilaxis con antimicrobianos así como el cambio frecuente de catéter y limitar su colocación a partir de las 22 semanas de embarazo (25). Otras complicaciones potenciales aunque mucho mas infrecuentes son la infección, migración y perforación $(2,13,25)$.

La nefrostomía percutánea, dirigida por ecografía, también ha demostrado ser un procedimiento de sencilla realización bajo anestesia local, proporcionando el drenaje espontáneo y la rápida recuperación de la función renal, permitiendo además el acceso para eventuales manipulaciones futuras y la instilación de sustancias para la disolución de ciertas litiasis $(13,26)$. La tasa de buenos resultados reportados con la nefrostomía es muy elevada (superior al $90 \%$ ) y presenta mejor coste-efectividad que el cateterismo (27). Sin embargo, sus posibles complicaciones (incrustación y obstrucción, infección, sangrado, descolocación del tubo de nefrostomía y dificultad técnica durante el tercer trimestre), la hacen desaconsejable especialmente cuando el término de la gestación no es cercano.

La ureteroscopia, tanto flexible como rígida, permite otra nueva posibilidad en el diagnóstico y tratamiento de la litiasis ureteral durante la gestación, estando además favorecida su realización como consecuencia de la dilatación fisiológica del uréter durante el embarazo, soliendo permitir que el ureteroscopio rígido pueda ser utilizado en la totalidad del tracto urinario, incluso durante estadios avanzados de gestación. Asimismo, el hecho de que el procedimiento pueda realizarse con anestesia local y sedación (28) lo convierten en una atractiva alternativa que permite fragmentar, extraer o desplazar cálculos así como colocar catéteres uréteres cuando fallan otras medidas menos agresivas.

\section{CONCLUSIONES}

En nuestra experiencia, la causa mas frecuente de hidronefrosis sintomática durante el embarazo la constituye la compresión ureteral por el útero grávido seguida de la etiología litiásica.

Consideramos improcedente la utilización de métodos de diagnóstico que empleen radiaciones, no sólo por los riesgos potenciales para el feto y por las implicaciones psicológicas para la paciente ante esta exposición (que suele rechazar), sino porque además, estas exploraciones no suelen aportar información que influya en la actitud terapéutica, ya que el diagnóstico etiológico y su tratamiento pueden ser habitualmente retrasados hasta la finalización del embarazo.

Con tratamiento conservador se suelen controlar con éxito la mayor parte de las pacientes independientemente de la causa subyacente. En los 
casos refractarios, tanto el cateterismo doble J como la nefrostomía percutánea han demostrado ser dos métodos seguros y eficaces, quedando en manos del urólogo la decisión de su empleo en función de las características clínicas y del momento de embarazo. Asimismo, la ureteroscopia constituye una nueva alternativa diagnóstica y terapéutica de la litiasis ureteral durante la gestación cuando fallan otras medidas menos agresivas.

\section{BIBLIOGRAFIA y LECTURAS RECOMENDADAS (*lectura de interés $y^{* *}$ lectura fundamental)}

*1. FAINARU, O.; ALMOG, B.; GAMZU, R. y cols.: "The management of symptomatic hydronephrosis in pregnancy". Br. J. Obstet. Gynaecol., 109: $1385,2002$.

2. ZWERGEL, T.; LINDENMEIR, T.; WULLICH, B.: "Management of acute hydronephrosis in pregnancy by ureteral stenting". Eur. Urol., 29: 292, 1996.

*3. BIYANI, C.S.; JOYCE, A.D.: "Urolithiasis in pregnancy. I: pathophysiology, fetal considerations and diagnosis". BJU Int., 89: 811, 2002.

4. IRVING, S.O.; BURGESS, N.A.: "Managing severe loin pain in pregnancy". Br. J. Obstet. Gynaecol., 109: 1025, 2002.

5. ECKFORD, S.D.; GINGELL, J.C.: "Ureteric obstruction in pregnancy-diagnosis and management". Br. J. Obstet. Gynaecol., 98: 1137, 1991.

**6. BAÑON, V.; RIGABERT, M.; NICOLAS, J. y cols.: "Manejo de la uropatía obstructiva en el embarazo". Actas Urol. Esp., 23: 227, 1999.

7. MAIKRANZ, P.; COE, F.L.; PARKS, J. y cols.: "Nephrolithiasis in pregnancy". Am. J. Kidney Dis., 9: 354, 1987.

8. MAIKRANZ, P.; HOLLEY, J.L.; PARKS, J.H. y cols.: "Gestational hypercalciuria causes pathological urine calcium oxalate supersaturations". Kidney Int., 36: 108, 1989.

9. PARULKAR, B.G.; HOPKINS, T.B.; WOLLIN, M.R. y cols.: "Renal colic during pregnancy: a case for conservative treatment". J. Urol., 159: $365,1998$.

10. STOTHERS, L.; LEE, L.M.: "Renal colic in pregnancy". J. Urol., 148: 1383, 1992.

11. SWANSON, S.K.; HEILMAN, R.L.; EVERSMAN, W.G.: "Urinary tract stones in pregnancy". Surg. Clin. North Am., 75: 123, 1995.

*12. GULMI, F.A.; FELPEN, D.; VAUGHAN, E.D.: "Fisiopatología de la obstrucción del tracto urina-

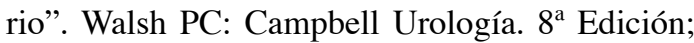
447-494; Ed Panamericana; Madrid, 2004.

13. BIYANI, C.S.; JOYCE, A.D.: "Urolithiasis in pregnancy. II: management”. BJU Int., 89: 819, 2002.
14. BRENT, R.L.: "The effect of embryonic and fetal exposure to $\mathrm{x}$-ray, microwaves, and ultrasound: counseling the pregnant and nonpregnant patient about these risks". Semin Oncol., 16: 347, 1989.

15. HELLAWELL, G.O.; COWAN, N.C.; HOLT, S.J.: "A radiation perspective for treating loin pain in pregnancy by double-pigtail stents". BJU Int., 90: 801, 2002.

16. MULLER-SUUR, R.; TYDEN, O.: "Evaluation of hydronephrosis in pregnancy using ultrasound and renography". Scand. J. Urol. Nephrol., 19: 267, 1985.

17. ASRAT, T.; ROOSIN, M.C.; MILLER, E.I.: "Ultrasonographic detection of ureteral jets in normal pregnancy”. Am. J. Obstet. Gynecol., 178: 1194, 1998.

18. DEYOE, L.A.; CRONAN, J.J.; BRESLAW, B.H. y cols.: "New techniques of ultrasound and color Doppler in the prospective evaluation of acute renal obstruction. Do they replace the intravenous urogram?". Abdom. Imaging., 20: 58, 1995.

19. BURKE, B.J.; WASHOWICH, T.L.: "Ureteral jets in normal second and third trimester pregnancy". J. Clin. Ultrasound, 26: 423, 1998.

20. LAING, F.C.; BENSON, C.B.; DISALVO, D.N. y cols.: "Distal ureteral calculi: detection with vaginal US". Radiology, 192: 545, 1994.

21. MACNEILY, A.E.; GOLDENBERG, S.L.; ALLEN, G.J. y cols.: "Sonographic visualization of the ureter in pregnancy". J. Urol., 146: 298, 1991.

22. OPDENAKKER, L.; OYEN, R.; VERVLOESSEM y cols.: "Acute obstruction of the renal collecting system: the intrarenal resistive index is a useful yet time-dependent parameter for diagnosis". Eur. Radiol., 8: 1429, 1998.

23. SPENCER, J.A.; CHAHAL, R.; KELLY, A. y cols.: "Evaluation of painful hydronephrosis in pregnancy: magnetic resonance urographic patterns in physiological dilatation versus calculous obstruction". J. Urol., 171: 256, 2004.

24. BRIGGS, G.G.; FREEMAN, R.K.; YAFFE, S.J.: "Drugs in pregnancy and lactation". $4^{\mathrm{a}}$ ed.; pp 6573; William and Wilkins, Baltimore, 1994.

25. DENSTEDT, J.D.; RAZVI, H.: "Management of urinary calculi during pregnancy". J. Urol., 148: 1072, 1992.

26. MOKHMALJI, H.; BRAUN, P.M.; MARTINEZ, F.J. y cols.: "Percutaneous nephrostomy versus ureteral stents for diversion of hydronephrosis caused by stones: a prospective, randomized clinical trial". J. Urol., 165: 1088, 2001.

27. PEARLE, M.S.; PIERCE, H.L.; MILLER, G.L. y cols.: "Optimal method of urgent decompression of the collecting system for obstruction and infection due to ureteral calculi". J. Urol., 160: 1260, 1998.

28. 28.- SHOKEIR, A.A.; MUTABAGANI, H.: "Rigid ureteroscopy in pregnant women". Br. J. Urol., 81: 678, 1998. 